OPEN ACCESS

Edited by:

Xiaofei $\mathrm{Hu}$

Army Medical University, China

Reviewed by:

Ling He,

Children's Hospital of Chongqing

Medical University, China

Yuting Zhang,

Children's Hospital of Chongqing

Medical University, China

*Correspondence:

Jingjing Su

jingjingsu2000@163.com

Fengchun Hua

huafc@hotmail.com

Xiaoxia Du

xxdu@phy.ecnu.edu.cn

tThese authors have contributed equally to this work and share first

authorship

Specialty section:

This article was submitted to

Applied Neuroimaging,

a section of the journal

Frontiers in Neurology

Received: 02 July 2021

Accepted: 08 October 2021

Published: 06 December 2021

Citation:

Li P, Huang Q, Ban S, Qiao Y, Wu J, Zhai Y, Du X, Hua F and Su J (2021)

Altered Default Mode Network Is

Associated With Cognitive Impairment

in CADASIL as Revealed by

Multimodal Neuroimaging.

Front. Neurol. 12:735033.

doi: $10.3389 /$ fneur.2021.735033

\section{Altered Default Mode Network Is Associated With Cognitive Impairment in CADASIL as Revealed by Multimodal Neuroimaging}

\author{
Panlong $\mathrm{Li}^{1 \dagger}$, Qi Huang ${ }^{2 \dagger}$, Shiyu Ban ${ }^{3}$, Yuan Qiao ${ }^{4}$, Jing $\mathrm{Wu}^{4}$, Yu Zhai ${ }^{4}$, Xiaoxia $\mathrm{Du}^{3 *}$, \\ Fengchun Hua ${ }^{5 *}$ and Jingjing $\mathrm{Su}^{4 *}$ \\ ${ }^{1}$ School of Electrical and Information Engineering, Zhengzhou University of Light Industry, Zhengzhou, China, ${ }^{2}$ Positron \\ Emission Tomography (PET) Center, Huashan Hospital, Fudan University, Shanghai, China, ${ }^{3}$ Shanghai Key Laboratory of \\ Magnetic Resonance and Department of Physics, School of Physics and Materials Science, East China Normal University, \\ Shanghai, China, ${ }^{4}$ Department of Neurology, Shanghai Ninth People's Hospital, Shanghai Jiao Tong University School of \\ Medicine, Shanghai, China, ${ }^{5}$ Department of Nuclear Medicine, Longhua Hospital, Shanghai University of Traditional Chinese \\ Medicine, Shanghai, China
}

Background and Purpose: Cerebral autosomal dominant arteriopathy with subcortical infarcts and leukoencephalopathy caused by mutations in the NOTCH3 gene is a hereditary cerebral small vessel disease, manifesting with stroke, cognitive impairment, and mood disturbances. Functional or structural changes in the default mode network $(\mathrm{DMN})$, which plays important role in cognitive and mental maintenance, have been found in several neurological and mental diseases. However, it remains unclear whether DMN is altered in patients with cerebral autosomal dominant arteriopathy with subcortical infarcts and leukoencephalopathy (CADASIL).

Methods: Multimodal imaging methods, including $\mathrm{MRI}$ and positron emission tomography (PET), were applied to evaluate the functional, structural, and metabolic characteristics of DMN in 25 patients with CADASIL and 42 healthy controls.

Results: Compared with controls, patients with CADASIL had decreased nodal efficiency and degree centrality of the dorsal medial pre-frontal cortex and hippocampal formation within DMN. Structural MRI and diffusion tensor imaging (DTI) showed decreased gray matter volume and fiber tracks presented in the bilateral hippocampal formation. Meanwhile, PET imaging showed decreased metabolism within the whole DMN in CADASIL. Furthermore, correlation analyses showed that these nodal characteristics, gray matter volume, and metabolic signals of DMN were related to cognitive scores in CADASIL.

Conclusions: Our results suggested that altered network characteristics of DMN might play important roles in cognitive deficits of CADASIL.

Keywords: CADASIL, default mode network, cognitive impairment, MRI, PET 


\section{INTRODUCTION}

Cerebral autosomal dominant arteriopathy with subcortical infarcts and leukoencephalopathy is the most common hereditary cerebrovascular disease (1). It is caused by the pathogenic mutations in the NOTCH3 gene on chromosome 19 and, therefore, shows familial inheritance. The typical clinical manifestations of cerebral autosomal dominant arteriopathy with subcortical infarcts and leukoencephalopathy (CADASIL) are migraine with aura, stroke, mood disturbances, and progressive cognitive impairments, including deficits in executive function, processing speed, attention, and memory (2).

Default mode network is a notable network that shows greater activity during the resting state than when performing tasks. It was first addressed by Raichle in a positron emission tomography (PET) study in 2001 (3). The component brain areas of default mode network (DMN) mainly include posterior cingulate cortex and precuneus (PCC/PCU), medial pre-frontal cortex (MPFC), medial and inferior temporal lobes, and inferior parietal lobe (IPL), and play important roles in a great variety of cognitive domains, such as working memory, visuomotor, visual language, and mental imagery (4). DMN has become a central research theme in neuropsychiatric disorders, including stroke, dementia, migraine, traumatic brain injury, depression, anxiety, and schizophrenia (5-10). A series of important research results about changed DMN characteristics in these disorders have been reported by using magnetic resonance imaging (MRI) and PET methods (5-10). However, as a focus of research into cognition, whether DMN is altered in patients with CADASIL remains unclear.

Several studies have demonstrated brain alterations in functional and structural imaging parameters in patients with CADASIL. Our previous resting-state functional MRI (fMRI) studies showed that altered functional activity and connectivity in PCC/PCU and para-hippocampal cortex (PHC) were associated with cognitive impairment in CADASIL $(11,12)$. Diffusion tensor imaging (DTI) studies have demonstrated widespread white matter lesions associated with cognitive deficits in CADASIL (13-15). Moreover, a case study using diffusion tensor tractography indicated that neural tract injuries were mainly located in the frontal lobe in a patient with CADASIL (16). Furthermore, a recent ${ }^{18} \mathrm{~F}$-2-fluoro-2-deoxy-D-glucose PET $\left({ }^{18} \mathrm{~F}\right.$ FDG PET) study showed that decreased metabolism in the limbic lobe, including the hippocampus and PHC, was positively associated with a cognitive score in patients with CADASIL (17). Several regions involved in CADASIL belong to the hub nodes of DMN, and the DMN has a high degree of connectivity across the above-involved regions, including the PCC/PCU, MPFC, hippocampus, and PHC $(5,18)$. Though promising initial results from different neuroimaging studies have shown considerable overlap with areas typically considered part of the DMN, few studies explored the DMN in patients with CADASIL. Thus, based on the brain regions highlighted in these CADASIL studies, we assumed DMN modifications in patients with CADASIL.

Therefore, in the present study, we focused on multimodal imaging outcomes of DMN in CADASIL. To characterize the DMN comprehensively, resting-state fMRI, T1-weighted MRI,
DTI, and ${ }^{18}$ F-FDG PET were employed to assess functional network properties, gray matter volume (GMV), structural connectivity, and metabolism in DMN, and the associations with cognitive deficits.

\section{MATERIALS AND METHODS}

\section{Participants}

The study protocol was approved by the ethics committee in the Shanghai Ninth People's Hospital. All participants were fully informed of the study procedures and signed the informed consent. A total of 25 patients with CADASIL from 14 families evaluated at Shanghai Ninth People's Hospital between May 2016 and January 2019 were recruited for this study. For all patients, the diagnosis was confirmed by the identification of pathogenic mutations in the NOTCH3 gene (19). All subjects underwent detailed standard neurological examinations. Subjects were excluded from the study if they had severe depression or anxiety according to evaluation by two trained neuropsychologists using the Hamilton Depression Scale (HAMD) and the Hamilton Anxiety Scale (HAMA) (20, 21). Subjects were diagnosed with severe depression and anxiety based on HAMD and HAMA scores $>17$ and $>14$, respectively. Neurological deficits in all subjects were assessed using the National Institutes of Health Stroke Scale (NIHSS) and the modified Rankin scale (mRs). Cognitive scores in all subjects were recorded by the Montreal Cognitive Assessment (MoCA) and Mini-Mental State Examination (MMSE). Most of the patients underwent both MRI and PET/computed tomography (PET/CT), but four underwent only MRI and another four underwent only PET/CT.

Forty-two healthy subjects were recruited as a control group based on the following criteria: no history of stroke, headache, cognitive impairment, or vascular disease risk factors; no family history of cerebrovascular diseases or vascular disease risk factors; not taking medications and no substance addiction, such as drugs, cigarettes, or alcohol. All of the healthy subjects had normal results on neurological and general examinations. Among the 42 controls, 21 underwent only MRI and the remaining 21 controls underwent only PET/CT. The sample size and demographic information of each group were listed in Table 1.

\section{MRI Acquisition}

Subjects in the first control group and 21 patients with CADASIL underwent MRI, including resting-state fMRI, structural MRI (T1-weighted, T2-weighted, and fluid-attenuated inversion recovery [FLAIR] imaging), and DTI on a 3 Tesla system (Trio Tim; Siemens Healthcare, Malvern, PA, USA) with a 12-channel head coil at East China Normal University. Soft earplugs and custom-fit foam were applied to reduce noise and movement artifacts. Resting-state fMRI was performed using a $\mathrm{T} 2{ }^{*}$-weighted gradient-echo echo-planar imaging pulse sequence with the following parameters: repetition time/echo time $(\mathrm{TR} / \mathrm{TE})=2,000 / 30 \mathrm{~ms}$; flip angle $=90^{\circ}$; field of view $(\mathrm{FOV})=220 \mathrm{~mm} \times 220 \mathrm{~mm}$; number of slices $=33$; resolution $=$ $3.44 \mathrm{~mm} \times 3.44 \mathrm{~mm} \times 4.38 \mathrm{~mm}$; total volume $=210$. The fMRI acquisition time was $7 \mathrm{~min}$ and $6 \mathrm{~s}$ for each subject. During the 
TABLE 1 | Demographic information of CADASIL and control.

\begin{tabular}{|c|c|c|c|c|c|c|}
\hline \multirow[b]{3}{*}{ Male/female } & \multirow{2}{*}{\multicolumn{2}{|c|}{$\begin{array}{c}\text { CADASIL }(n=25) \\
\text { MRI }(n=21), \text { PET }(n=21)\end{array}$}} & \multirow{2}{*}{\multicolumn{2}{|c|}{$\begin{array}{c}\text { Controls }(n=42) \\
\text { MRI }(n=21) \text {, PET }(n=21)\end{array}$}} & \multicolumn{2}{|c|}{$p$-values } \\
\hline & & & & & \multirow{2}{*}{$\begin{array}{c}\text { p1 } \\
1\end{array}$} & \multirow{2}{*}{$\begin{array}{c}p 2 \\
1\end{array}$} \\
\hline & $13 / 8$ & $14 / 7$ & $13 / 8$ & $14 / 7$ & & \\
\hline Age (years), mean \pm SD & $48.4 \pm 14.2$ & $46.3 \pm 14.0$ & $48.7 \pm 14.3$ & $45.8 \pm 12.1$ & 0.9 & 0.9 \\
\hline Education (years), mean \pm SD & $8.7 \pm 3.5$ & $8.9 \pm 3.3$ & $9.1 \pm 3.2$ & $9.3 \pm 3.0$ & 0.8 & 0.8 \\
\hline Family history, $n$ (\%) & $20(95.2)$ & $21(100)$ & - & - & - & - \\
\hline Migraine, $n(\%)$ & $3(14.3)$ & $3(14.3)$ & - & - & - & - \\
\hline Migraine with aura, $n(\%)$ & $2(9.5)$ & $2(9.5)$ & - & - & - & - \\
\hline Migraine without aura, $n(\%)$ & $1(4.8)$ & $1(4.8)$ & - & - & - & - \\
\hline WMH volume $\left(\mathrm{cm}^{3}\right)$, mean $\pm \mathrm{SD}(n=19)$ & $78.3 \pm 55.7$ & - & - & - & - & - \\
\hline Lacunar volume $\left(\mathrm{cm}^{3}\right)$, mean $\pm \mathrm{SD}(n=17)$ & $1.9 \pm 1.8$ & - & - & - & - & - \\
\hline Microbleeds (number), mean \pm SD $(n=6)$ & $6.0 \pm 1.8$ & - & - & - & - & - \\
\hline O'Sullivan sign, $n$ (\%) & 9 (42.9) & & & & & \\
\hline HAMD, median $\pm I Q R$ & $4 \pm 6$ & $4 \pm 5$ & $3 \pm 4$ & $3 \pm 3$ & 0.3 & 0.3 \\
\hline HAMA, median \pm IQR & $3 \pm 1$ & $3 \pm 1$ & $3 \pm 1$ & $3 \pm 2$ & 0.7 & 0.7 \\
\hline NIHSS, median \pm IQR & $0 \pm 1$ & $0 \pm 1$ & $0 \pm 0$ & $0 \pm 0$ & 0.01 & 0.006 \\
\hline mRs, median $\pm I Q R$ & $1 \pm 2$ & $1 \pm 2$ & $0 \pm 0$ & $0 \pm 0$ & 0.001 & 0.001 \\
\hline MoCA, median \pm IQR & $21 \pm 15$ & $20 \pm 11$ & $26 \pm 3$ & $27 \pm 1$ & 0.000 & 0.000 \\
\hline MMSE, median \pm IQR & $24 \pm 12$ & $24 \pm 11$ & $28 \pm 1$ & $28 \pm 2$ & 0.001 & 0.002 \\
\hline
\end{tabular}

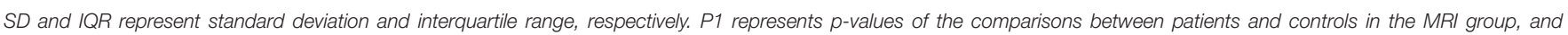

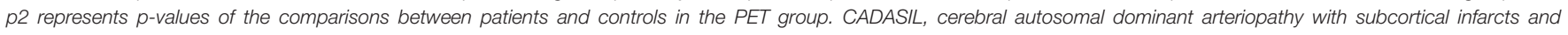

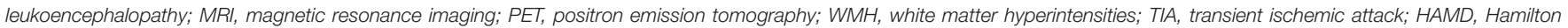

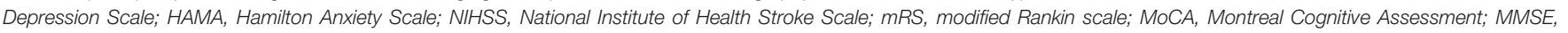
Mini-Mental State Examination.

fMRI scan, the subjects kept their eyes closed but did not fall asleep. The whole-brain anatomical volume was obtained using a high-resolution T1-weighted 3D magnetization-prepared rapidacquisition gradient-echo pulse sequence with the following parameters: $\mathrm{TR}=2,530 \mathrm{~ms}$; $\mathrm{TE}=2.34 \mathrm{~ms}$; flip angle $=7^{\circ} ; \mathrm{FOV}=$ $256 \mathrm{~mm} \times 256 \mathrm{~mm}$; number of slices $=192$; resolution $=1 \mathrm{~mm}$ $\times 1 \mathrm{~mm} \times 1 \mathrm{~mm}$. The T1-weighted image acquisition time was $6 \mathrm{~min}$ and $3 \mathrm{~s}$ for each subject. T2-weighted imaging was obtained using turbo spin-echo dark fluid sequence with the following parameters: $\mathrm{TR} / \mathrm{TE}=5,500 / 83 \mathrm{~ms} ; \mathrm{FOV}=220 \mathrm{~mm} \times 220 \mathrm{~mm}$; number of slices $=35$; resolution $=0.38 \mathrm{~mm} \times 0.38 \mathrm{~mm} \times$ $5.2 \mathrm{~mm}$. The T2-weighted image scanning time was $1 \mathrm{~min}$ and $26 \mathrm{~s}$ for each subject. The parameters of FLAIR imaging were: $\mathrm{TR} / \mathrm{TE}=9,000 / 93 \mathrm{~ms} ; \mathrm{FOV}=220 \mathrm{~mm} \times 220 \mathrm{~mm}$; number of slices $=30$; resolution $=0.43 \mathrm{~mm} \times 0.43 \mathrm{~mm} \times 4.55 \mathrm{~mm}$. The FLAIR image scanning time was $4 \mathrm{~min}$ and $50 \mathrm{~s}$ for each subject. DTI was performed using a single-shot, spin-echo planar imaging sequence acquired in contiguous axial planes with the following parameters: 64 non-collinear directions, diffusion weighting of $b$ $=1,000 \mathrm{~s} / \mathrm{m}^{2}$, an acquisition without diffusion weighting of $\mathrm{b}=$ 0 ; $\mathrm{TR} / \mathrm{TE}=8,900 / 86 \mathrm{~ms} ; \mathrm{FOV}=256 \mathrm{~mm} \times 256 \mathrm{~mm}$, covered the whole brain; 70 contiguous slices; resolution $=2 \mathrm{~mm} \times 2 \mathrm{~mm}$ $\times 2 \mathrm{~mm}$. The DTI scanning time was $10 \mathrm{~min}$ and $7 \mathrm{~s}$ for each subject. The total MRI acquisition time was $29 \mathrm{~min}$ and $38 \mathrm{~s}$ for each subject.

\section{PET Acquisition}

${ }^{18}$ F-2-fluoro-2-deoxy-D-glucose PET data were acquired using a Siemens Biograph Truepoint HD 64 PET/CT is made by Simens in Germany at the PET Center of Huashan Hospital, Fudan University. ${ }^{18}$ F-FDG was synthesized and radiolabeled at the PET Center according to the protocol of the manufacturer under the inspection by the Chinese Food and Drug Administration. Before ${ }^{18}$ F-FDG injection, subjects were asked to avoid strenuous physical activity and fast for about $6 \mathrm{~h}$ to maintain blood glucose level $<8 \mathrm{mmol} / \mathrm{L}$. After receiving an injection of ${ }^{18} \mathrm{~F}-\mathrm{FDG}$ at a dose of $5.55 \mathrm{MBq} / \mathrm{kg}(0.15 \mathrm{mCi} / \mathrm{kg})$, subjects rested in a dimly lit room for $50 \mathrm{~min}$. Before PET acquisition, a low-dose CT scan was performed for attenuation correction, and then 10-min PET images were reconstructed using a filtered back-projection algorithm. The matrix size of the reconstructed images was 168 $\times 168 \times 148$ with a resolution of $2.04 \mathrm{~mm} \times 2.04 \mathrm{~mm} \times 1.5 \mathrm{~mm}$.

\section{Data Processing}

Prior to preprocessing, all the raw DICOM data were converted to the Neuroimaging Informatics Technology Initiative format (NII) using MRICRON software (https://people.cas.sc.edu/ rorden/mricron/install.html) and the quality of the images was checked visually.

\section{fMRI Data Processing}

The resting-state fMRI data were preprocessed using Data Processing Assistant for Resting-State fMRI (DPARSF; http:// www.restfmri.net) $(22,23)$. Data were preprocessed starting with the removal of the first 10 volumes to ameliorate possible effects of scanner instability and the adaptation of subjects to the environment. Then, slice time correction was applied to reduce the effects of within-scan acquisition time differences between 


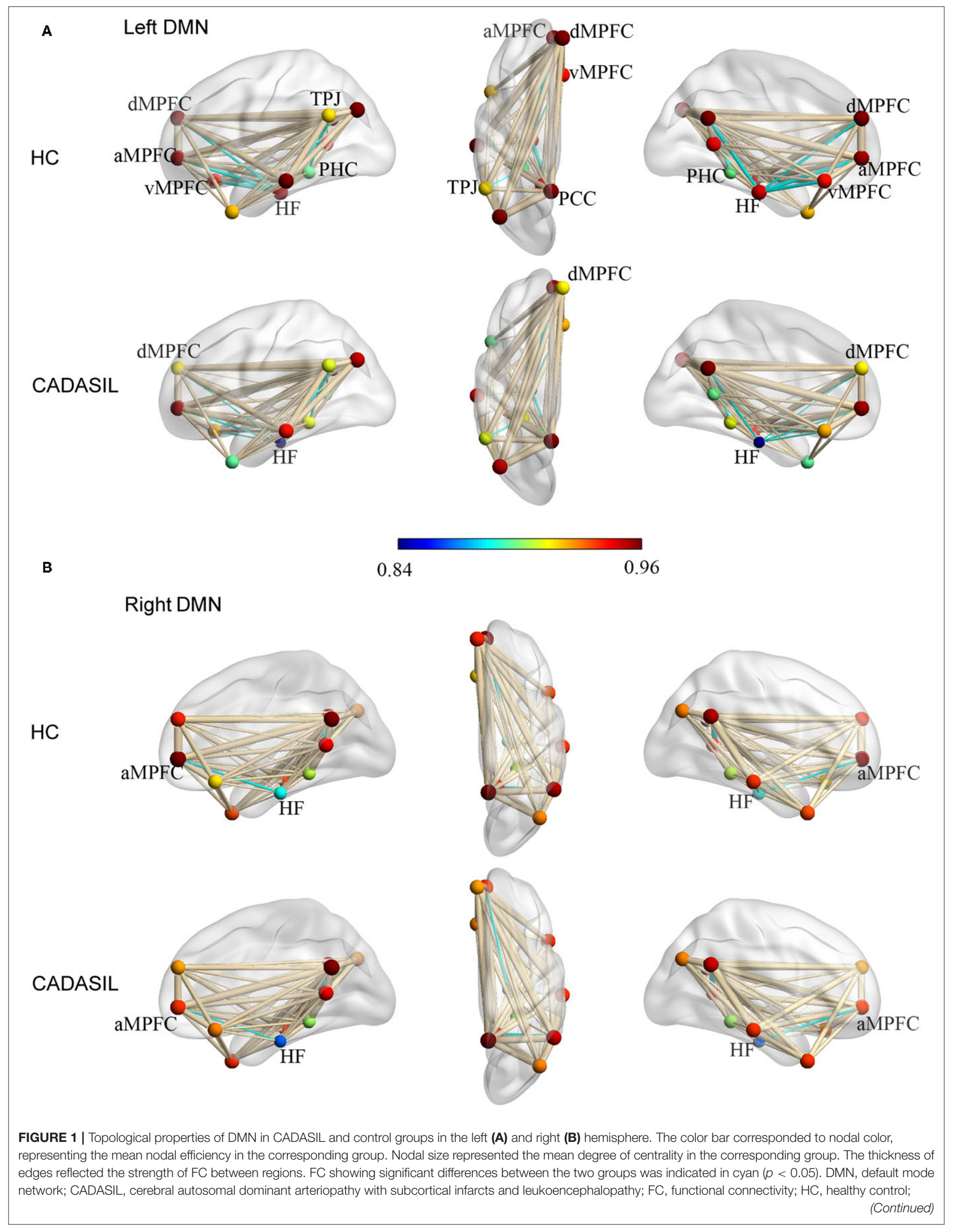


FIGURE 1 | a MPFC, anterior medial pre-frontal cortex; dMPFC, dorsal medial pre-frontal cortex; VMPFC, ventral medial pre-frontal cortex; HF, hippocampal formation; PCC, posterior cingulate cortex; PHC, parahippocampal cortex; TPJ, temporal-parietal junction.

slices. To correct the effects of head motion, the fMRI images of each subject were realigned and registered. All subjects had head motions $<1.5^{\circ}$ of rotation or $0.5 \mathrm{~mm}$ of mean framewise displacement (24). The fMRI images were then normalized into the Montreal Neurological Institute (MNI) space using the EPI template and smoothed by a full-width at half-maximum (FWHM) $8 \mathrm{~mm}$ Gaussian kernel. Following spatial smoothing, linear detrend was performed to remove noise due to longterm physiological shifts, movement-related noise remaining after realignment, and instrumental instability. To reduce further the effects of noise, the fMRI images were filtered with a temporal band-pass filter $(0.01-0.08 \mathrm{~Hz})$. Finally, the six head motion parameters, global mean signal, white matter signal, and cerebrospinal fluid signal were regressed out as nuisance covariates to remove these unwanted signals.

With reference to previous studies $(18,25,26), 11$ separate regions comprising the left $\mathrm{DMN}$ and 11 mirrored regions comprising the right DMN were defined as regions of interest (ROIs). The 11 ROIs were spheres of radius $8 \mathrm{~mm}$ in the dorsal MPFC (dMPFC), anterior MPFC (aMPFC), ventral MPFC (vMPFC), posterior IPL (pIPL), temporal-parietal junction (TPJ), lateral temporal cortex (LTC), temporal pole (TempP), PCC, retrosplenial cortex (RSC), PHC, and hippocampal formation (HF) (see Supplementary Table 1 and Supplementary Figure 1 for coordinates and spatial positions). Average fMRI time-series were calculated across every voxel in each ROI. The absolute value of Fisher's z-transformed Pearson's correlation coefficient between each pair of time-series was defined as the functional connectivity (FC) strength.

Graph analysis of the pairwise $(11 \times 11)$ correlation matrixes was performed using GRETNA (v2.0.0; https://www. nitrc.org/projects/gretna/) (27). Global and nodal network properties, including nodal degree centrality, nodal shortest path length, nodal clustering coefficient, nodal efficiency, nodal local efficiency, betweenness centrality, global efficiency, assortativity coefficient, and small-worldness, were calculated to delineate the integrative and local topological architecture of the DMN, respectively. Their definitions and calculations of the nodal and global network properties were summarized in Supplementary Table 2.

\section{T1-Weighted Data Processing}

The T1-weighted MRI data were preprocessed using the Computational Anatomy Toolbox (CAT12; http://dbm.neuro. uni-jena.de/cat12) implemented in statistical parametric mapping software (SPM12; htttp://www.fil.ion.ucl.ac.uk/spm/). First, all T1-weighted MRI data were normalized into the MNI space using the Diffeomorphic Anatomic Registration Through Exponentiated Lie algebra algorithm (DARTEL). The bias field inhomogeneities were corrected to remove non-uniform intensities. Normalized images were then segmented into gray matter, white matter, and cerebrospinal fluid components. The total intracranial volume (TIV) of each participant was evaluated to correct for the effects of differences in brain size. The internal gray matter threshold was set to 0.2 to exclude artifacts on the gray-white matter border. Thereafter, all preprocessed scans were smoothed with the FWHM $6 \mathrm{~mm}$ Gaussian kernel. Finally, the average GMV was calculated across every voxel in each ROI.

\section{DTI Data Processing}

The raw DTI data were preprocessed using FMRIB Software Library (FSL; http://www.fmrib.ox.ac.uk/fsl/index.html.) First, eddy current correction was performed to correct for head motion artifacts and eddy current distortions. Then, the brain of each subject was extracted using the FSL Brain Extraction Tool (BET). Tensor reconstruction and fiber tracking were applied by Diffusion Toolkit TrackVis (https://www.nitrc.org/projects/ trackvis.) The Fiber Association Continuous Tracking (FACT) algorithm in Diffusion Toolkit was applied to obtain the wholebrain fiber tracts. The main parameters in fiber tractography were as follows: maximum turning angle threshold at $35^{\circ}$; minimum fractional anisotropy (FA) threshold of 0.2. Then, SPM12 was applied to bring all the individual tracts into the MNI space by non-linear transformation methods. In the normalization step, tracts were spatially normalized by: co-registering T1-weighted MRI to the corresponding FA image; calculating the deformation field of the individual coregistered T1-weighted image space to the MNI space; applying the deformation field to tracts and bringing them into the MNI space. Thereafter, TrackVis was used to record the number of tracts (NT) passing through each ROI.

\section{PET Data Processing}

First, PET images of each subject were processed using SPM12 software with spatial normalization and smoothing. The PET template in SPM12 was used in the spatial normalization step. The FWHM $8 \mathrm{~mm}$ Gaussian kernel was applied in the smoothing step. The average glucose metabolism was then calculated across every voxel in each ROI.

\section{Statistical Analysis}

Statistical analysis was performed using IBM SPSS Statistics for Windows (SPSS, Chicago, IL, USA). The Chi-square tests and permutation tests (permutation times $=10,000$ ) were used to compare demographic, clinical, and imaging characteristics between the CADASIL and control groups, as appropriate. Furthermore, the two-sample $t$-test was applied for voxel-wise metabolism comparisons between the CADASIL and corresponding control groups using SPM12 software. Subsequently, partial correlations were established to estimate the relations between the cognitive deficits and the imaging characteristics showing significant between-group differences. Age, sex, and education levels were entered as covariates in partial correlation analysis. Benjamini-Hochberg false discovery rate (FDR) correction was further used to avoid type-I errors in 
A

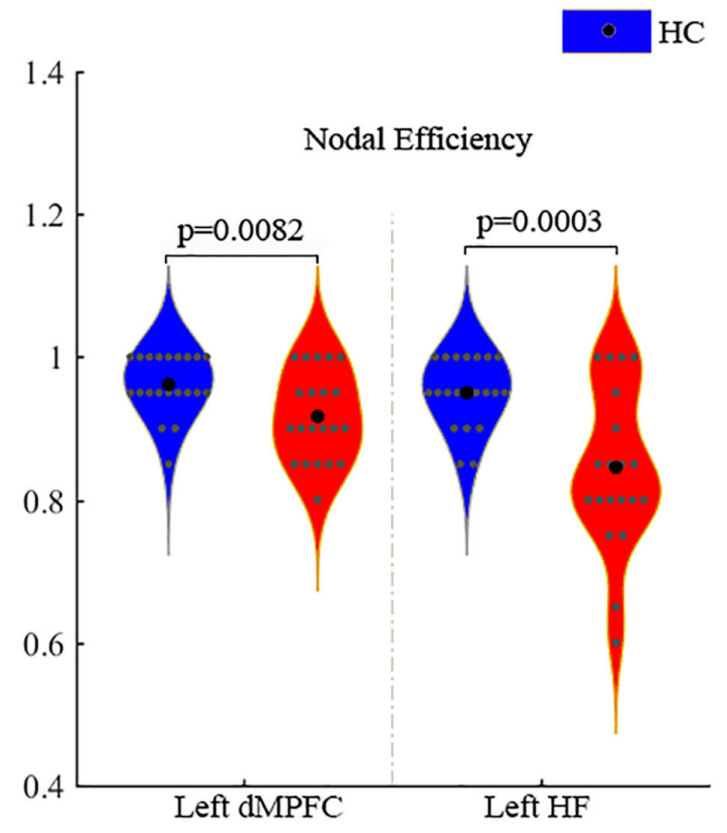

B
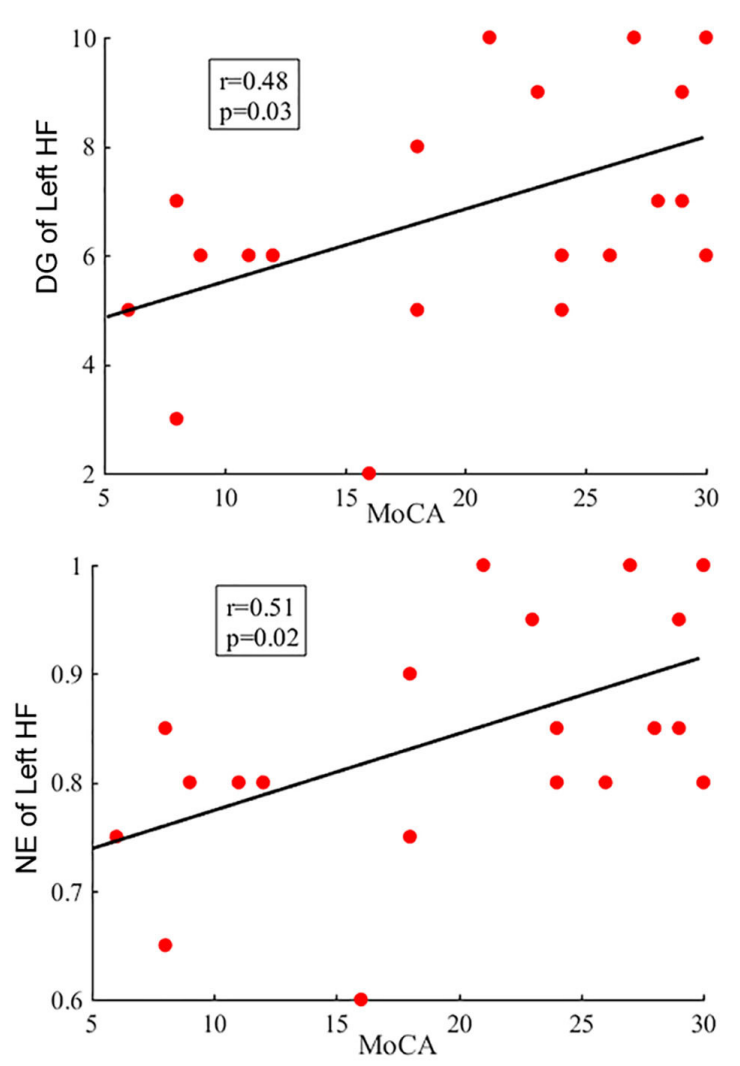

- CADASIL
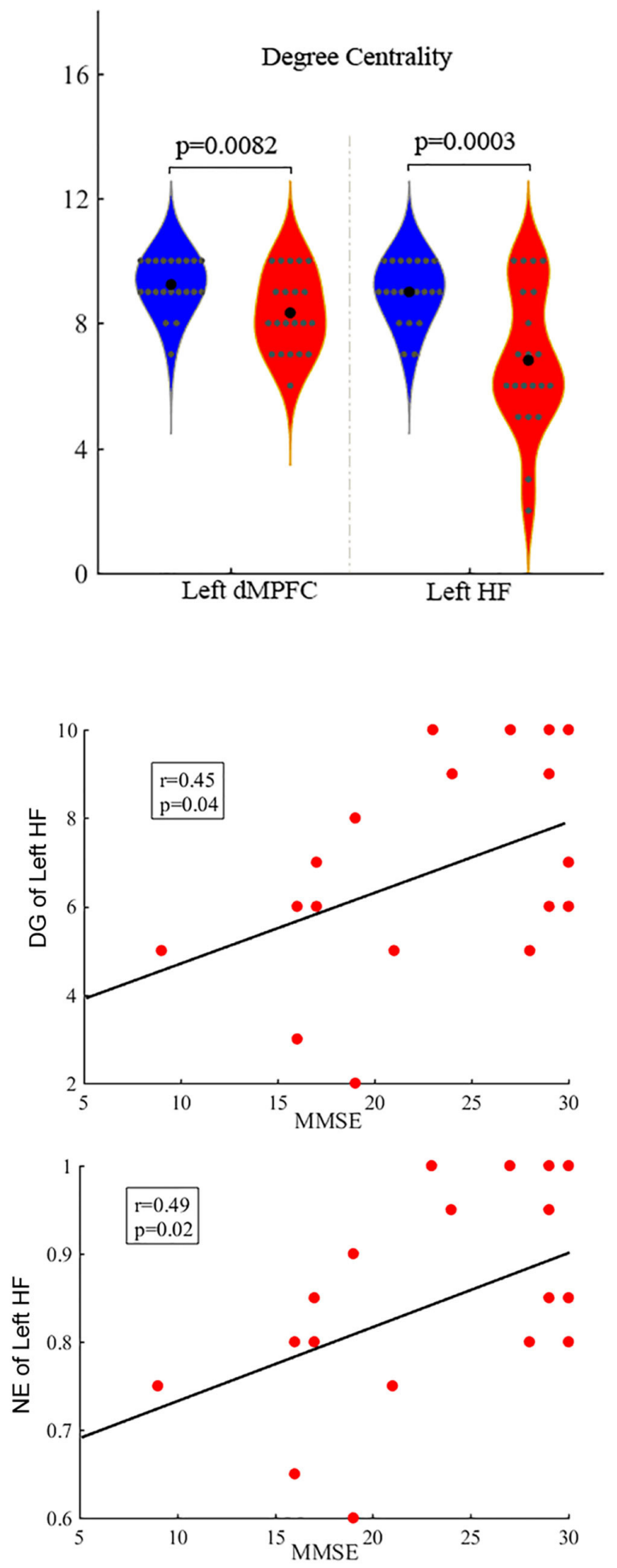

FIGURE 2 | Differences between CADASIL and control groups in topological properties and correlations between the properties and cognitive scores in the DMN. (A) Nodal efficiency and degree centrality were significantly different between the CADASIL and healthy control groups in the left DMN. The CADASIL had decreased nodal efficiency and degree centrality in the left dMPFC and HF. (B) Linear correlations between network properties in the DMN and cognitive scores in CADASIL. 
FIGURE 2 | CADASIL, cerebral autosomal dominant arteriopathy with subcortical infarcts and leukoencephalopathy; DMN, default mode network; dMPFC, dorsal medial pre-frontal cortex; HF, hippocampal formation; HC, healthy control; DG, degree centrality; NE, nodal efficiency; MoCA, Montreal Cognitive Assessment; MMSE, Mini-Mental State Examination.

the multiple comparisons and correlations. The results of twosample comparisons and partial correlations were regarded as significant at $p<0.05$ (two-tailed) with FDR correction.

\section{RESULTS}

\section{Demographic and Clinical Data}

Table 1 showed comparisons of the demographic and clinical data of the CADASIL and healthy control groups. There were significant differences between the two groups in terms of neurological deficits and cognitive scores, but no differences in sex, age, education levels, or depression and anxiety symptom scores.

\section{Network Analysis of DMN}

Compared with the healthy control group, the CADASIL group had decreased FC between the HF and the MPFC (aMPFC, dMPFC, and vMPFC), as well as increased FC between the TPJ and PHC within the left DMN (Figure 1A and Supplementary Figure 2). Further network analysis showed that the nodal characteristics of the left dMPFC and HF, including nodal efficiency and degree centrality, were significantly different between the CADASIL and healthy control groups (Figure 2A). The integrative topological architecture of the left DMN was not significantly different between the two groups.

In the right DMN, the CADASIL group showed decreased FC between the HF and the aMPFC as well as the AMPFC, and increased FC between the TPJ and RSC in comparison to the healthy control group (Figure 1B and Supplementary Figure 2). There were no significant differences in the global or local topological architecture of the right DMN between the two groups.

\section{GMV Analysis of DMN}

There was no significant difference in TIV between the CADASIL group and the healthy control group. Compared with the healthy control group, the patients with CADASIL had decreased GMV in the left PHC $(0.58 \pm 0.1$ vs. $0.66 \pm 0.12$, respectively, $p=0.02)$ and bilateral HF (left: $0.38 \pm 0.04$ vs. $0.41 \pm 0.05$, respectively, $p$ $=0.035$; right: $0.40 \pm 0.05$ vs. $0.44 \pm 0.06$, respectively, $p=0.04$ ) (Figure 3A).

\section{Fiber Tracks Analysis of DMN}

Compared with the healthy control group, the patients with CADASIL had reduced NT in the bilateral HF (left: $198.86 \pm$ 85.86 vs. $263.33 \pm 64.18$, respectively, $p=0.008$; right: 208.05 \pm 72.68 vs. $290.24 \pm 84.01$, respectively, $p=0.001$ ) (Figure 4). There was no significant increase in tracks between the CADASIL and healthy control groups.

\section{Metabolism Analysis of DMN}

Compared with the healthy control group, the patients with CADASIL had decreased glucose metabolism across the ROIs $(p<0.00001)$ (Figure 5A). There was a significant decrease in global metabolism across the whole brain $(p<0.00001)$ (Figure 5A). There was no significant increase in regional metabolism between the two groups.

\section{Correlation Analysis}

In fMRI, the nodal efficiency and degree centrality in the bilateral HF were positively correlated with MoCA and MMSE scores in the patients with CADASIL $(p<0.05)$ (Figure 2B). Similar correlations between the GMV of the bilateral HF and left PHC, and cognitive scores were also detected in the CADASIL group (Figure 3B). There was no significant correlation between the number of tracks and cognitive scores. The levels of metabolism in most ROIs with decreased glucose metabolism were positively correlated with the cognitive scores in the CADASIL group $(p<$ 0.01) (Figure 5B and Supplementary Table 3).

\section{DISCUSSION}

The present study was performed to investigate whether the DMN was altered in patients with CADASIL. We integrated the results of multimodal imaging methods, including fMRI, T1-weighted MRI, DTI, and PET, to investigate the changes in functional network properties, GMV, fiber tracks, and glucose metabolism within the DMN in patients with CADASIL. Consistent with our initial hypothesis, the characteristics of DMN represented by these images in the CADASIL group were significantly different compared with those in the healthy control group. Correlation analysis showed that these modifications were associated with cognitive deficits in the CADASIL group. These findings had important implications for the further understanding of CADASIL-related cognitive deficits and could provide potential brain markers for CADASIL.

Previous neuroimaging studies have focused on the whole brain functional or structural changes in CADASIL by a single imaging modality $(15,17,28)$. In our study, we focused on the DMN by multi-modal imaging methods. The results in this study corresponded with previous MRI studies on stroke, dementia, migraine, and WMH that showed that functional or structural modifications of DMN are associated with cognitive deficits. In this study, more than $90 \%$ of CADASIL subjects had WMH. Although the ratio of migraine, dementia, and migraine was not as high as WMH, these symptoms are also typical of the later clinical manifestations of CADASIL. Notably, in order to fully and thoroughly explore the DMN alterations in CADASIL, except for three MRI modalities, one PET modality which represents the glucose metabolism in the brain was applied to evaluate the network characteristics of DMN. In order to reduce the influence 

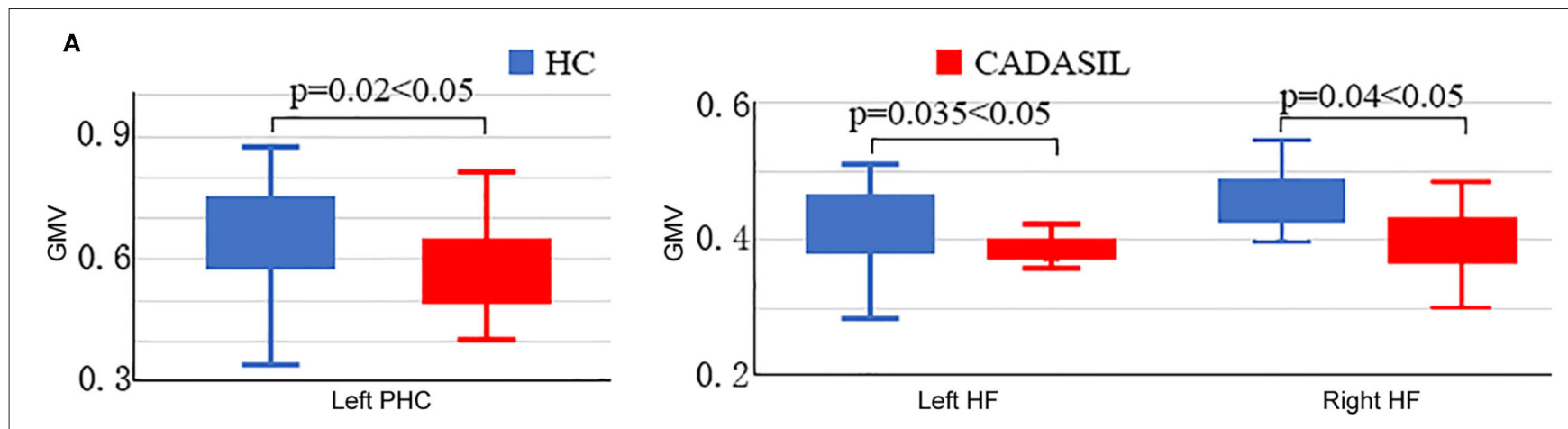

B
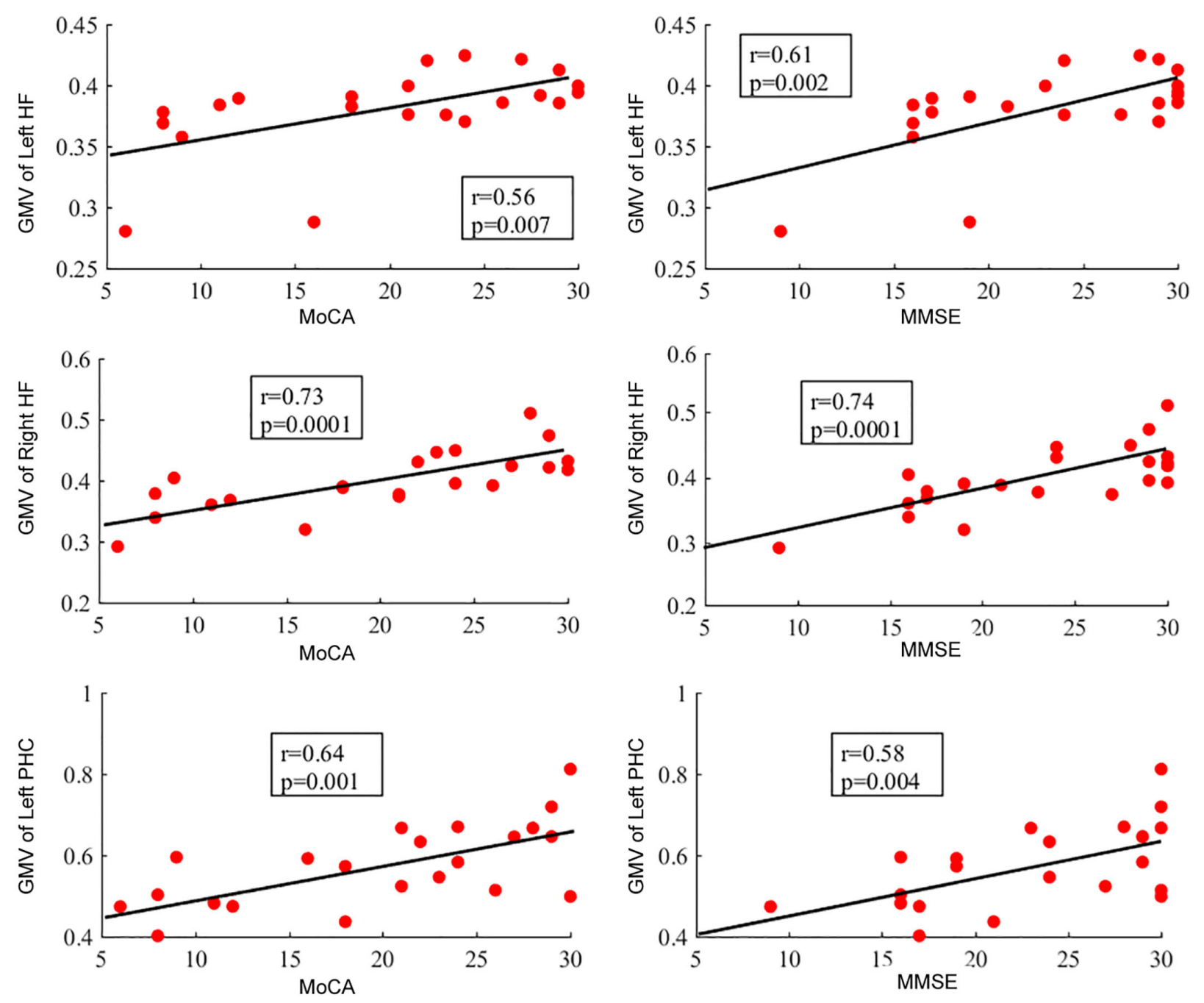

FIGURE 3 | Results of GMV analysis in CADASIL and control groups. (A) Alterations in GMV of the DMN in CADASIL. Patients with CADASIL had decreased GMV in the left PHC and bilateral HF compared with healthy controls. (B) Correlations between GMV of ROls within the DMN and cognitive scores in CADASIL. GMV, gray matter volume; CADASIL, cerebral autosomal dominant arteriopathy with subcortical infarcts and leukoencephalopathy; DMN, default mode network; PHC, parahippocampal cortex; HF, hippocampal formation; ROI, region of interest; $\mathrm{HC}$, healthy control; MoCA, Montreal Cognitive Assessment; MMSE, Mini-Mental State Examination. 


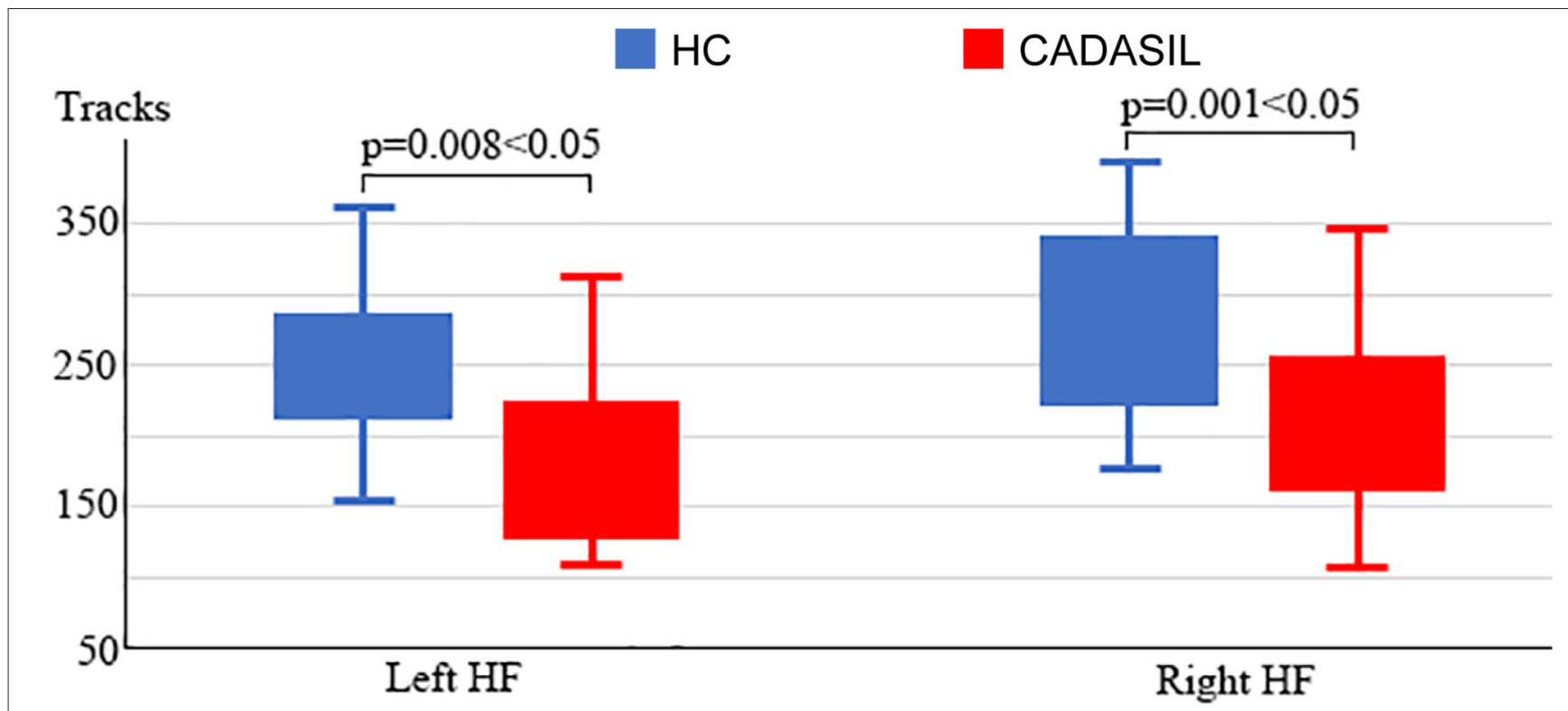

FIGURE 4 | Analysis of fiber tracks in the DMN in CADASIL and control groups. Patients with CADASIL had decreased fiber tracks in the bilateral HF. DMN, default mode network; CADASIL, cerebral autosomal dominant arteriopathy with subcortical infarcts and leukoencephalopathy; HF, hippocampal formation; HC, healthy control.
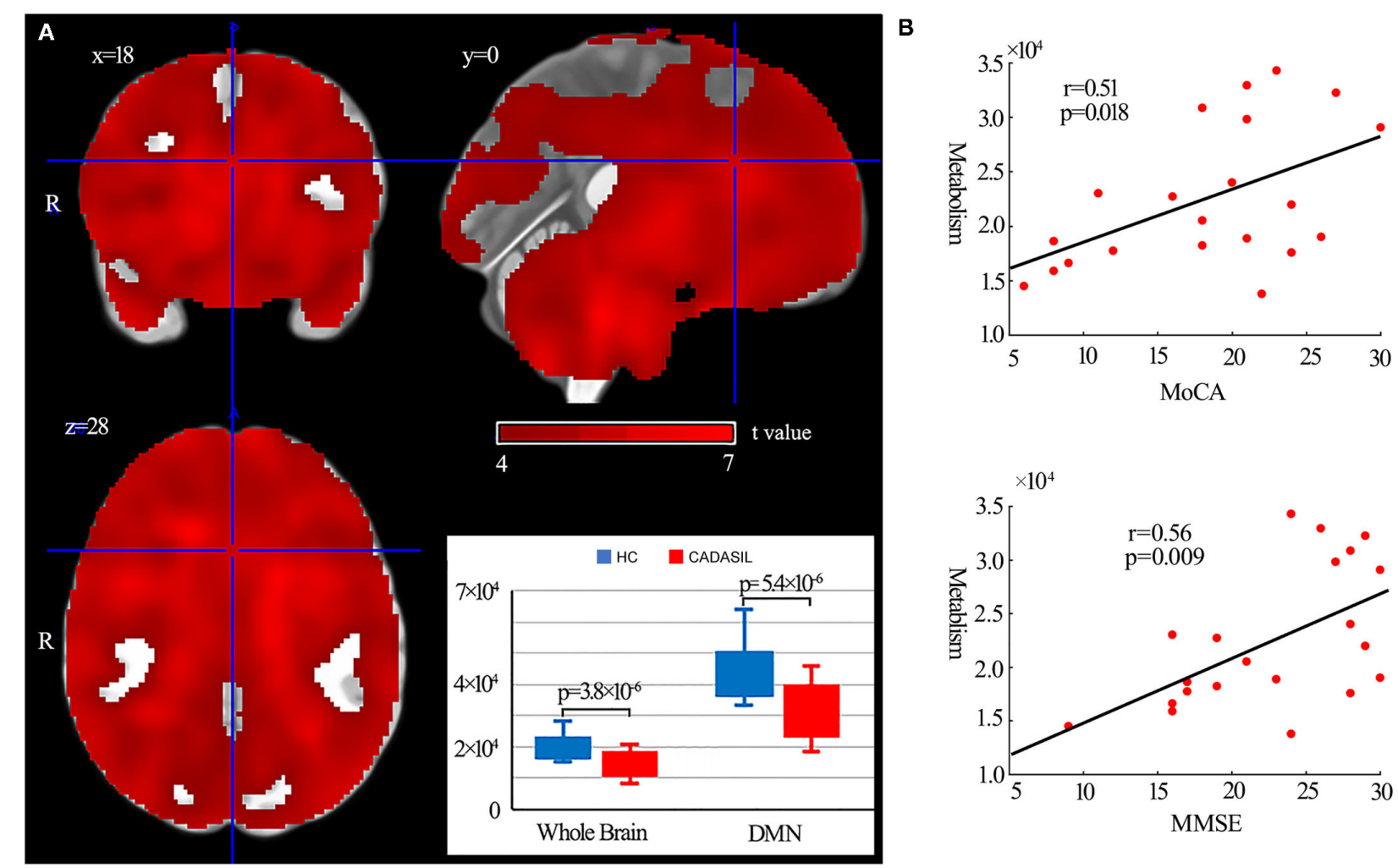

FIGURE 5 | Results of metabolism analysis in the DMN in CADASIL and control groups. (A) Metabolism alterations in the CADASIL group. (B) Correlations between metabolism across ROls within the DMN and cognitive scores in the CADASIL group. Patients with CADASIL had decreased metabolism across the whole brain, including the ROls within the DMN, compared with healthy controls. DMN, default mode network; CADASIL, cerebral autosomal dominant arteriopathy with subcortical infarcts and leukoencephalopathy; ROI, region of interest; HC, healthy control; MoCA, Montreal Cognitive Assessment; MMSE, Mini-Mental State Examination. 
of different subjects on the results, most of the patients with CADASIL (17 of 21) had both PET and MRI modalities.

In the FC analysis, weakened interactions between the HF and MPFC (aMPFC and AMPFC) were detected in both the left and right DMN in patients with CADASIL. Further graph theory analysis of the FC matrixes showed that the changed FC resulted in decreased nodal efficiency and degree centrality of HF and dMPFC in the CADASIL group. These observations indicated that HF and dMPFC within the DMN in the patients with CADASIL have a poor capacity for information propagation, and reduced functional interactions with other regions within the network. Indeed, the HF plays a central node in memory function (29-31) and the dMPFC plays a key role in cognitive performance, including decision making, reward processing, mentalizing, memory, and conceptual processing $(6,31,32)$. In addition, functional interactions between the HF and MPFC have been demonstrated to form an important neural circuit for spatial working memory (33-35). Furthermore, functional alterations in the HF or MPFC have been shown to be associated with cognitive deficits in other diseases (36-40). Therefore, our fMRI results demonstrated that the changed FC strength as a particular locus of dysfunction affected the nodal properties of the DMN, which may contribute to cognitive deficits in patients with CADASIL.

To examine structural changes within the DMN in CADASIL, differences between the two groups in GMV and NT of the ROIs were examined. Analyses of both the GMV and NT showed that patients with CADASIL had decreased GMV and NT in the ROI of HF. Further, decreased GMV was found in the left PHC and the bilateral HF in the CADASIL group. Both the PHC and HF are key regions for memory-related cognition (31, 41, 42). In addition, decreased GMV as well as changed FA in the two regions have been reported to be associated with cognitive deficits $(15,43-45)$. The significant associations between the GMV of HF and cognitive scores were consistent with the results of a previous MRI study in a large cohort (46). The HF belongs to the medial temporal subsystem of the DMN, which, through its interactions with the MPFC, plays a role in a wide range of associative or constructive aspects of mental simulation (6). Indeed, decreased interactions between the MPFC and medial temporal lobe including the hippocampus in restingstate fMRI data, and decreased GMV in the two regions, have been suggested to be associated with cognitive deficits, including working memory, social and emotional processing, and executive function deficits $(39,40,43-45,47,48)$. The two regions have been suggested to play hub roles in DMN, which is a hub network for advanced cognition $(18,25)$. Cognitive performance relies on the coordination and collaboration of the activation and deactivation response; if one component fails, the whole system is jeopardized $(49,50)$. Therefore, the overlap between the results of fMRI and structural MRI indicates that changes of HF within the DMN may play important roles in the cognitive deficits seen in patients with CADASIL, and HF may be a potential brain marker. These MRI results corresponded with a previous DMN study in Fabry disease that showed functional DMN modifications and white matter damage were associated with cognitive deficits (51). However, contrary to the pattern of reduced DMN FC and decreased GMV found in our results, there were no significant differences in GMV, and increased DMN FC was observed in patients with Fabry disease. These differences between CADASIL and Fabry disease implied different pathogenic mechanisms underlying the two conditions, which can be further investigated. In marked contrast to the local variation in MRI results, ${ }^{18} \mathrm{~F}$-FDG PET showed that glucose metabolism of each ROI within the whole DMN, and even the whole brain, was decreased in the CADASIL group. Although there have been few PET studies in CADASIL, two independent PET studies reported hypometabolism across the whole brain in the resting state in patients with CADASIL $(52,53)$, which was consistent with our findings. Furthermore, similar to the results of MRI, metabolism was positively correlated with the cognitive scores in the CADASIL group. These correlations between characteristics of DMN revealed by different imaging modalities and cognitive scores suggested that interior relations may underlie the functional and structural changes within the DMN in patients with CADASIL. However, regional functional or structural disconnection can affect metabolism in other regions and vice versa. Further longitudinal multimodal imaging studies are required to determine the underlying mechanisms.

This study has some limitations, as only resting-state fMRI or PET data were collected, and it is, therefore, unclear whether there were altered patterns of DMN activity or connectivity during task performance in CADASIL, which had been demonstrated in other disorders or with aging (54-57).

\section{CONCLUSIONS}

In conclusion, altered functional and structural properties of the DMN were found in patients with CADASIL by multimodal imaging. These cognition-associated changes of HF within the DMN may play important roles in cognitive deficits in CADASIL.

\section{DATA AVAILABILITY STATEMENT}

The raw data supporting the conclusions of this article will be made available by the authors, without undue reservation.

\section{ETHICS STATEMENT}

The studies involving human participants were reviewed and approved by Independent Ethics Committee of Shanghai Ninth Hospital, Shanghai Jiao Tong University School of Medicine. The patients/participants provided their written informed consent to participate in this study.

\section{AUTHOR CONTRIBUTIONS}

JS, FH, and XD: study conception and design. PL, QH, JW, YQ, and YZ: acquisition of data. PL and SB: analysis and interpretation of data. All authors 
drafting the article and final approval of the version to be published.

\section{FUNDING}

This research was supported by grants from the National Natural Science Foundation of China (82071282 to JS), the Rare Disease Registration Platform of Shanghai Ninth People's Hospital, Shanghai Jiao Tong University School of Medicine (JYHJB08 to JS), and the Horizontal Research Project from Shanghai Ninth People's Hospital (JYHX2021001 to JS).

\section{REFERENCES}

1. Di Donato I, Bianchi S, De Stefano N, Dichgans M, Dotti MT, Duering M, et al. Cerebral autosomal dominant arteriopathy with subcortical infarcts and leukoencephalopathy (CADASIL) as a model of small vessel disease: update on clinical, diagnostic, and management aspects. BMC Med. (2017) 15:41. doi: 10.1186/s12916-017-0778-8

2. Davous P. CADASIL: a review with proposed diagnostic criteria. Eur J Neurol. (1998) 5:219-33. doi: 10.1046/j.1468-1331.1998.530219.x

3. Raichle ME, MacLeod AM, Snyder AZ, Power WJ, Gusnard DA, Shulman GL. A default mode of brain function. Proc Natl Acad Sci USA. (2001) 98:676-82. doi: 10.1073/pnas.98.2.676

4. Hu ML, Zong XF, Mann JJ, Zheng JJ, Liao YH, Li ZC, et al. a review of the functional and anatomical default mode network in schizophrenia. Neurosci Bull. (2017) 33:73-84. doi: 10.1007/s12264-016-0090-1

5. Menon V. Large-scale brain networks and psychopathology: a unifying triple network model. Trends Cogn Sci. (2011) 15:483-506. doi: 10.1016/j.tics.2011.08.003

6. Andrews-Hanna JR, Smallwood J, Spreng RN. The default network and self-generated thought: component processes, dynamic control, and clinical relevance. Ann N Y Acad Sci. (2014) 1316:29-52. doi: 10.1111/nyas.12360

7. Rodriguez-Cano E, Alonso-Lana S, Sarro S, Fernandez-Corcuera P, Goikolea JM, Vieta E, et al. Differential failure to deactivate the default mode network in unipolar and bipolar depression. Bipolar Disord. (2017) 19:38695. doi: 10.1111/bdi.12517

8. Messina R, Filippi M, Goadsby PJ. Recent advances in headache neuroimaging. Curr Opin Neurol. (2018) 31:37985. doi: 10.1097/WCO.0000000000000573

9. Guo X, Duan X, Suckling J, Chen H, Liao W, Cui Q, et al. Partially impaired functional connectivity states between right anterior insula and default mode network in autism spectrum disorder. Hum Brain Mapp. (2019) 40:126475. doi: $10.1002 / \mathrm{hbm} .24447$

10. Zong X, Hu M, Pantazatos SP, Mann JJ, Wang G, Liao Y, et al. A dissociation in effects of risperidone monotherapy on functional and anatomical connectivity within the default mode network. Schizophr Bull. (2019) 45:1309-18. doi: 10.1093/schbul/sby175

11. Su J, Ban S, Wang M, Hua F, Wang L, Cheng X, et al. Reduced resting-state brain functional network connectivity and poor regional homogeneity in patients with CADASIL. J Headache Pain. (2019) 20:103. doi: 10.1186/s10194-019-1052-6

12. Su J, Wang M, Ban S, Wang L, Cheng X, Hua F, et al. Relationship between changes in resting-state spontaneous brain activity and cognitive impairment in patients with CADASIL. J Headache Pain. (2019) 20:36. doi: 10.1186/s10194-019-0982-3

13. Molko N, Pappata S, Mangin JF, Poupon F, LeBihan D, Bousser MG, et al. Monitoring disease progression in CADASIL with diffusion magnetic resonance imaging: a study with whole brain histogram analysis. Stroke. (2002) 33:2902-8. doi: 10.1161/01.STR.0000041681.25514.22

14. Viswanathan A, Godin O, Jouvent E, O'Sullivan M, Gschwendtner A, Peters N, et al. Impact of MRI markers in subcortical vascular dementia: a multi-modal analysis in CADASIL. Neurobiol Aging. (2010) 31:162936. doi: 10.1016/j.neurobiolaging.2008.09.001

\section{ACKNOWLEDGMENTS}

We would like to gratefully thank the patients and healthy volunteers taking part in this study.

\section{SUPPLEMENTARY MATERIAL}

The Supplementary Material for this article can be found online at: https://www.frontiersin.org/articles/10.3389/fneur. 2021.735033/full\#supplementary-material

15. Ban S, Wang H, Wang M, Xu S, Qin Z, Su J, et al. Diffuse tract damage in CADASIL is correlated with global cognitive impairment. Eur Neurol. (2019) 81:294-301. doi: 10.1159/000501612

16. Jang $\mathrm{SH}$, Seo YS. Injuries of neural tracts in a patient with CADASIL: a diffusion tensor imaging study. BMC Neurol. (2015) 15:176. doi: 10.1186/s12883-015-0434-x

17. Su J, Huang Q, Ren S, Xie F, Zhai Y, Guan Y, et al. Altered brain glucose metabolism assessed by F-FDG PET imaging is associated with the cognitive impairment of CADASIL. Neuroscience. (2019) 417:3544. doi: 10.1016/j.neuroscience.2019.07.048

18. Andrews-Hanna JR, Reidler JS, Sepulcre J, Poulin R, Buckner RL. Functionalanatomic fractionation of the brain's default network. Neuron. (2010) 65:550 62. doi: 10.1016/j.neuron.2010.02.005

19. Joutel A, Corpechot C, Ducros A, Vahedi K, Chabriat H, Mouton P, et al. Nothc3 mutations in CADASIL, a hereditary adult-onset condition causing stroke and dementia. Nature. (1996) 383:707-10. doi: 10.1038/383707a0

20. Mozen-Zadeh E, Bayanati S, Ziafat K, Rezaei F, Mesgarpour B, Akhondzadeh S. Vortioxetine as adjunctive therapy to risperidone for treatment of patients with chronic schizophrenia: a randomised, doubleblind, placebo-controlled clinical trial. Journal of Psychopharm. (2020) 34:506-13. doi: 10.1177/0269881120909416

21. Zimmerman M, Thompson JS, Diehl JM, Balling C, Kiefer R. Is the DSM-5 Anxious Distress Specifier Interview a valid measure of anxiety in patients with generalized anxiety disorder: a comparison to the Hamilton Anxiety Scale. Psychiatry Res. (2020) 286:112859. doi: 10.1016/j.psychres.2020.112859

22. Chao-Gan Y, Yu-Feng Z. DPARSF: A MATLAB Toolbox for "Pipeline" data analysis of resting-state fMRI. Front Syst Neurosci. (2010) 4:13. doi: $10.3389 /$ fnsys. 2010.00013

23. Yan CG, Wang XD, Zuo XN, Zang YF. DPABI: Data processing \& analysis for (Resting-State) brain imaging. Neuroinformatics. (2016) 14:33951. doi: 10.1007/s12021-016-9299-4

24. Power JD, Barnes KA, Snyder AZ, Schlaggar BL, Petersen SE. Spurious but systematic correlations in functional connectivity MRI networks arise from subject motion. Neuroimage. (2012) 59:2142-54. doi: 10.1016/j.neuroimage.2011.10.018

25. Buckner RL, Andrews-Hanna JR, Schacter DL. The brain's default network: anatomy, function, and relevance to disease. Ann N Y Acad Sci. (2008) 1124:1-38. doi: 10.1196/annals.1440.011

26. Buckner RL, Sepulcre J, Talukdar T, Krienen FM, Liu H, Hedden T, et al. Cortical hubs revealed by intrinsic functional connectivity: mapping, assessment of stability, and relation to Alzheimer's disease. J Neurosci. (2009) 29:1860-73. doi: 10.1523/JNEUROSCI.5062-08.2009

27. Wang J, Wang X, Xia M, Liao X, Evans A, He Y. GRETNA: a graph theoretical network analysis toolbox for imaging connectomics. Front Hum Neurosci. (2015) 9:386. doi: 10.3389/fnhum.2015.00458

28. Pan H, Huang Q, Ban S, Du X, Su J, Liu J. Brain structural changes in CADASIL patients: a morphometric magnetic resonance imaging study. Neurosci Lett. (2020) 738:135388. doi: 10.1016/j.neulet.2020.135388

29. Eichenbaum H. Does the hippocampus preplay memories? Nat Neurosci. (2015) 18:1701-2. doi: 10.1038/nn.4180

30. Bird CM. The role of the hippocampus in recognition memory. Cortex. (2017) 93:155-65. doi: 10.1016/j.cortex.2017.05.016 
31. Hainmueller T, Bartos M. Parallel emergence of stable and dynamic memory engrams in the hippocampus. Nature. (2018) 558:292-6. doi: 10.1038/s41586-018-0191-2

32. Lv C, Wang Q, Chen C, Xue G, He Q. Activation patterns of the dorsal medial prefrontal cortex and frontal pole predict individual differences in decision impulsivity. Brain Imaging Behav. (2020) 15:4219. doi: 10.1007/s11682-020-00270-1

33. Benchenane K, Peyrache A, Khamassi M, Tierney PL, Gioanni Y, Battaglia FP, et al. Coherent theta oscillations and reorganization of spike timing in the hippocampal- prefrontal network upon learning. Neuron. (2010) 66:92136. doi: 10.1016/j.neuron.2010.05.013

34. Hyman JM, Zilli EA, Paley AM, Hasselmo ME. Working memory performance correlates with prefrontal-hippocampal theta interactions but not with prefrontal neuron firing rates. Front Integr Neurosci. (2010) 4:2. doi: 10.3389/neuro.07.002.2010

35. Wirt RA, Hyman JM. integrating spatial working memory and remote memory: interactions between the medial prefrontal cortex and hippocampus. Brain Sci. (2017) 7:43. doi: 10.3390/brainsci7040043

36. Moran JM, Jolly E, Mitchell JP. Social-cognitive deficits in normal aging. $J$ Neurosci. (2012) 32:5553-61. doi: 10.1523/JNEUROSCI.5511-11.2012

37. Pasquini L, Scherr M, Tahmasian M, Meng C, Myers NE, Ortner M, et al. Link between hippocampus' raised local and eased global intrinsic connectivity in AD. Alzheimers Dement. (2015) 11:475-84. doi: 10.1016/j.jalz.2014.02.007

38. Zhu L, Shu H, Liu D, Guo Q, Wang Z, Zhang Z. Apolipoprotein E epsilon4 specifically modulates the hippocampus functional connectivity network in patients with amnestic mild cognitive impairment. Front Aging Neurosci. (2018) 10:289. doi: 10.3389/fnagi.2018.00289

39. Tao J, Liu J, Chen X, Xia R, Li M, Huang M, et al. Mind-body exercise improves cognitive function and modulates the function and structure of the hippocampus and anterior cingulate cortex in patients with mild cognitive impairment. Neuroimage Clin. (2019) 23:101834. doi: 10.1016/j.nicl.2019.101834

40. Liang L, Zhao L, Wei Y, Mai W, Duan G, Su J, et al. Structural and functional hippocampal changes in subjective cognitive decline from the community. Front Aging Neurosci. (2020) 12:64. doi: 10.3389/fnagi.2020.00064

41. Brown TI, Stern CE. Contributions of medial temporal lobe and striatal memory systems to learning and retrieving overlapping spatial memories. Cereb Cortex. (2014) 24:1906-22. doi: 10.1093/cercor/bht041

42. Eichenbaum H. On the integration of space, time, and memory. Neuron. (2017) 95:1007-18. doi: 10.1016/j.neuron.2017.06.036

43. Chen B, Fan GG, Liu H, Wang S. Changes in anatomical and functional connectivity of Parkinson's disease patients according to cognitive status. Eur J Radiol. (2015) 84:1318-24. doi: 10.1016/j.ejrad.2015.04.014

44. Konishi K, McKenzie S, Etchamendy N, Roy S, Bohbot VD. Hippocampus-dependent spatial learning is associated with higher global cognition among healthy older adults. Neuropsychologia. (2017) 106:310-21. doi: 10.1016/j.neuropsychologia.2017.09.025

45. Jirsaraie RJ, Sheffield JM, Barch DM. Neural correlates of global and specific cognitive deficits in schizophrenia. Schizophr Res. (2018) 201:23742. doi: 10.1016/j.schres.2018.06.017

46. O'Sullivan M, Ngo E, Viswanathan A, Jouvent E, Gschwendtner A, Saemann PG, et al. Hippocampal volume is an independent predictor of cognitive performance in CADASIL. Neurobiol Aging. (2009) 30:8907. doi: 10.1016/j.neurobiolaging.2007.09.002

47. Wang L, Zang Y, He Y, Liang M, Zhang X, Tian L, et al. Changes in hippocampal connectivity in the early stages of Alzheimer's disease: evidence from resting state fMRI. Neuroimage. (2006) 31:496-504. doi: 10.1016/j.neuroimage.2005.12.033
48. Kennedy DP, Courchesne E. The intrinsic functional organization of the brain is altered in autism. Neuroimage. (2008) 39:187785. doi: 10.1016/j.neuroimage.2007.10.052

49. Wermke M, Sorg C, Wohlschlager AM, Drzezga A. A new integrative model of cerebral activation, deactivation and default mode function in Alzheimer's disease. Eur J Nucl Med Mol Imaging. (2008) 35(Suppl. 1):S1224. doi: 10.1007/s00259-007-0698-5

50. Broyd SJ, Demanuele C, Debener S, Helps SK, James CJ, Sonuga-Barke EJ. Default-mode brain dysfunction in mental disorders: a systematic review. Neurosci Biobehav Rev. (2009) 33:279-96. doi: 10.1016/j.neubiorev.2008. 09.002

51. Cocozza S, Pontillo G, Quarantelli M, Sacca F, Riccio E, Costabile $\mathrm{T}$, et al. Default mode network modifications in Fabry disease: a resting-state fMRI study with structural correlations. Hum Brain Mapp. (2018) 39:1755-64. doi: 10.1002/hbm 23949

52. Tatsch K, Koch W, Linke R, Poepperl G, Peters N, Holtmannspoetter M, et al. Cortical hypometabolism and crossed cerebellar diaschisis suggest subcortically induced disconnection in CADASIL: An ${ }^{18}$ F-FDG PET study. J Nuclear Med. (2003) 44:862-9.

53. Tuominen S, Miao Q, Kurki T, Tuisku S, Pöyhönen M, Kalimo H, et al. Positron emission tomography examination of cerebral blood flow and glucose metabolism in young CADASIL patients. Stroke. (2004) 35:10637. doi: 10.1161/01.STR.0000124124.69842.2d

54. Kennedy DP, Redcay E, Courchesne E. Falling to deactivate: Resting functional abnormalities in autism. Proc Nat Acad Sci USA. (2006) 103:8275-80. doi: 10.1073/pnas.06006 74103

55. Andrews-Hanna JR, Snyder AZ, Vincent JL, Lustig C, Head D, Raichle ME, et al. Disruption of large-scale brain systems in advanced aging. Neuron. (2007) 56:924-35. doi: 10.1016/j.neuron.2007.10.038

56. Li LM, Violante IR, Leech R, Ross E, Hampshire A, Opitz A, et al. Brain state and polarity dependent modulation of brain networks by transcranial direct current stimulation. Hum Brain Mapp. (2018) 40:90415. doi: $10.1002 / \mathrm{hbm} .24420$

57. Hughes C, Faskowitz J, Cassidy BS, Sporns O, Krendl AC. Aging relates to a disproportionately weaker functional architecture of brain networks during rest and task states. Neuroimage. (2020) 209:116521. doi: 10.1016/j.neuroimage.2020.116521

Conflict of Interest: The authors declare that the research was conducted in the absence of any commercial or financial relationships that could be construed as a potential conflict of interest.

Publisher's Note: All claims expressed in this article are solely those of the authors and do not necessarily represent those of their affiliated organizations, or those of the publisher, the editors and the reviewers. Any product that may be evaluated in this article, or claim that may be made by its manufacturer, is not guaranteed or endorsed by the publisher.

Copyright $\odot 2021$ Li, Huang, Ban, Qiao, Wu, Zhai, Du, Hua and Su. This is an open-access article distributed under the terms of the Creative Commons Attribution License (CC BY). The use, distribution or reproduction in other forums is permitted, provided the original author(s) and the copyright owner(s) are credited and that the original publication in this journal is cited, in accordance with accepted academic practice. No use, distribution or reproduction is permitted which does not comply with these terms. 